\title{
TOP QUARK MASS AND MIXINGS IN THE PRESENCE OF A HEAVY QUARK FAMILY
}

\author{
Carl H. ALBRIGHT* \\ CERN - Geneva
}

\begin{abstract}
The top quark mass and mixings are sludied in the framework of an extended Fritzsch model for purposes of illustration, where the fourth-family of heavy quarks either form a standard doublet or exist simply as isosinglets. We have fixed the masses of the heavy quarks near the infrared fixed point, unlike previous studies which select the top quark mass in a narrow range and then try to predict the heavy quark masses. The models are satisfactory with a top mass near $70 \mathrm{GeV}$ and suggest that the appearance of a top quark mass significantly lower than $90 \mathrm{GeV}$, as favored by three quark families in previous studies, can serve as a strong hint of new physics beyond the standard model.
\end{abstract}

*Permanent address: Department of Physics, Northern Illinois University, DeKalb, lltinois 60115

CERN-TH.5343/89

March 1989 
In a recent series of papers, ${ }^{1-4}$ the author, in collaboration carly on with Jarlskog and Lindholm and more recently with hindner, has explored in some detail three family quark mass matrices in the weak basis and their predictions for the KM mixing matrix, $B-\bar{B}$ mixing, $\left|V_{u b}\right| /\left|V_{c b}\right|$, the bag parameter in $\mathrm{K}$ decay and other accessible experimental quantities of interest. The mass matrices which appear to give the best fits to all the known dala are those of Fritzsch ${ }^{5}$ based on hicrarchical chiral symmetry breaking and modifications thereof suggested by Lindner and the author. ${ }^{4}$ The standard Fritzsch model is satisfactory ${ }^{2}$ only in the presence of two Higgs doublets, while the model suggested in ref. 4 also works with the minimal Iliggs structure. Corrections have also been given $n^{3,4}$ to account for mass matrix evolution from a high chiral symmel,y-brcaking scale $\sim 100 \mathrm{TeV}$, for example, down to the 1 GeV scalc, due to nonlinear effects introduced by Iliggs exchange graphs. The outcome of this study is that the present known data can be fit in the three quark framework, provided the top quark mass is approximately $90 \mathrm{GeV}$. Although many other authors ${ }^{6}$ have also looked into this problem, our approach through the invariant operator trace technique of Jarlskog, ${ }^{7}$ along with inclusion of nonlinear evolution effects, has enabled us to present, rather precise results for each model in a graphically clear fashion.

In this letter we proceed to study the case of four quark families. Atthough the literature on four standard doublet families is also rather extensive, ${ }^{8}$ we adopt a different approach. In place of setting the top quark mass in some range like 30 - $60 \mathrm{GeV}$ to find the masses of the fourth-family inembers, we shall fix the heavy fourth-family masses near the infrared fixed point, to study the restrictions on the top quark mass and mixings. Of even more inlerest are the recent suggestions ${ }^{9}$ that, in the framework of an $S U(2)_{L} \times S U(2)_{R} \times U(1)_{Y}$ theory, a family of isoscalar quarks or mirror quarks can become massive al, some higher scale and, in turn, generate 
masses for the standard three quark families by a secsaw mechanism and radiative corrections. These models lead nalurally to flavor-changing neutral currents in tree level. Given the apparently excellent results previously obtained for threc quark families and the possibility that a fourth quark family actually exists, the question arises whether the new family effectively decouples from the other three, or whether suitable mixings can be generated also in accord with present data. If the latifer is the case, we would naturally guess that the predictions for the top quark mass would be substantially altered. We shall show that satisfactory mixing results can be obtained, now with the somewhat lower value of $m_{t} \simeq 70 \mathrm{GcV}$. This present study remains incomplete in that the rather complex evolution effects have not yet been calculated, but we have tried to minimize those effects for purposes of illustration by restricting the choice of fourth-family masses so as to present these very timely results as soon as possible. The evolution delails will be presented elscwhere, along with a more comprehensive selection of fourth family masses.

For the purposes of this initial study and to reduce the complexity as much as possible, we shall assume that mixings with the fourth family do occur but adopt. the simple nearest-neighbor Fritzsch form of the mass matrices in the weak basis as extended to four families:

$$
\mathbf{M}^{U}=\left(\begin{array}{cccc}
0 & A & 0 & 0 \\
A & 0 & B & 0 \\
0 & B & 0 & D \\
0 & 0 & D^{*} & E
\end{array}\right), \quad \mathbf{M}^{D}=\left(\begin{array}{cccc}
0 & A^{\prime} & 0 & 0 \\
A^{\prime *} & 0 & B^{\prime} & 0 \\
0 & B^{\prime *} & 0 & D^{\prime} \\
0 & 0 & D^{\prime *} & E^{\prime}
\end{array}\right)
$$

This implies that the $t$ and $b$ quarks get massive al the sccond stage of chiral symmetry breaking via a scesaw mechanism and/or radiative corrections. We have eliminated the unphysical phases by a diagonal phase rotation, so that 12 parametcrs are available to predict the 17 independent physical parameters (8 masses +6 
mixing angles and 3 phases of the extended KM matrix). For these IIermitian mass matrices, the 8 magnitude parameters can be evaluated from the invariant traces $\operatorname{Tr} \mathrm{M}, \operatorname{Tr} \mathrm{M}^{2}, \operatorname{Tr} \mathrm{M}^{3}$ and determinant, Det $\mathrm{M}$, where we identify the diagonal mass matrices in the mass eigenbases as

$$
\mathbf{D}^{U}=\operatorname{diag}\left(m_{u},-m_{c}, m_{t},-m_{X}\right), \quad \mathbf{D}^{D}=\operatorname{diag}\left(m_{d},-m_{s}, m_{b},-m_{Y}\right)
$$

with the signs chosen to satisfy positivity requirements for $|D|^{2}$, etc. We are then left with just $\phi_{D}, \phi_{A^{\prime}}, \phi_{B^{\prime}}$ and $\phi_{D^{\prime}}$ to explain the 9 extended $\mathrm{KM}$ mixing angles and phases. Of course, $m_{t}, m_{X}$ and $m_{Y}$ are unknown; we fix $m_{X}^{\text {phys }}=250 \mathrm{GeV}$ and $m_{Y}^{\text {phys }}=240 \mathrm{GeV}$, which are values associaled with the infrared fixed points, a theoretically appealing choice and one which minimizes the nonlinear effects of evolution ${ }^{10}$ from the $\Lambda_{S B} \sim 100 \mathrm{TeV}$ scale down to $1 \mathrm{GcV}$ and minimizes, as well, the radiative corrections to the $\rho$ parameter. ${ }^{11}$

The $4 \times 4$ unitary matrices $U$ and $U^{\prime}$ which diagonalize the Hermitian matrices in (1a) satisfy

$$
U \mathbf{M}^{U} U^{\dagger}=\mathbf{D}^{U}, \quad U^{\prime} \mathbf{M}^{D} U^{\prime \dagger}=\mathbf{D}^{D}
$$

and relate the weak and mass cigenstates with four standard doublet familics by

$$
\begin{aligned}
& \psi^{U}=\left(u^{\prime}, c^{\prime}, t^{\prime}, X^{\prime}\right)^{T}=U^{\dagger} \Psi^{U}=U^{\dagger}(u, c, t, X)^{T} \\
& \psi^{D}=\left(d^{\prime}, s^{\prime}, b^{\prime}, Y^{\prime}\right)^{T}=U^{\prime \dagger} \Psi^{D}=U^{\prime \dagger}(d, s, b, Y)^{T}
\end{aligned}
$$

so the extended $4 \times 4$ unitary $\mathrm{KM}$ matrix is just the matrix product

$$
\left(\mathrm{V}_{K M}\right)_{\alpha \beta}=U_{\alpha \gamma}\left(U^{\dagger \dagger}\right)_{\gamma \beta} ; \quad \alpha, \beta, \gamma=1,2,3,4
$$

However, in the case where the fourth family is isosinglet and only three quark families participate in the $S U(2)_{L}$ charged current weak interactions, the three pairs of weak eigenstates are given by $\psi_{1,2,3}^{U}=u^{\prime}, c^{\prime}, t^{\prime}$ and $\psi_{1,2,3}^{D}=d^{\prime}, s^{\prime}, b^{\prime}$, 
respectively. The fourth combinations, $\psi_{4}^{U}=X^{\prime}$ and $\psi_{4}^{D}=Y^{\prime}$, are the irrelevant weak isoscalars. In this latter case, the extended $4 \times 1 \mathrm{KM}$ matrix is nonunitary and given by

$$
\left(\mathcal{V}_{K M}\right)_{\alpha \beta}=U_{\alpha i}\left(U^{\prime \dagger}\right)_{i \beta} ; \quad \alpha, \beta=1,2,3,4 ; \quad i=1,2,3
$$

in terms of the product of $4 \times 3$ and $3 \times 4$ malrices. In either case, the "observed" KM matrix is

$$
\left(V_{K M}^{o b s}\right)_{i j}=\left(V_{K M}\right)_{i j} ; \quad i, j=1,2,3
$$

or

$$
\left(V_{K M}^{\text {obs }}\right)_{i j}=\left(\mathcal{V}_{K M}\right)_{i j} ; \quad i, j=1,2,3
$$

respectively.

For comparison with the experimental information on the mixing matrix, we use the recent analysis of Schubert ${ }^{12}$ for three quark families described by the mixing matrix

$$
\left(\left|\mathbf{V}_{i j}\right|\right)=\left(\begin{array}{lll}
0.9754 \pm 0.0004 & 0.2206 \pm 0.0018 & 0.0000+0.0123 \\
0.2203 \pm 0.0019 & 0.9743 \pm 0.0005 & 0.0460 \pm 0.0060 \\
0.0101 \pm 0.0122 & 0.0419 \pm 0.0065 & 0.9989 \pm 0.0003
\end{array}\right)
$$

but impose only the constraints on $V_{u d}, V_{u s}, V_{c d}$ and $V_{c b}$ in our extension to four quark families. The other entries have becn considerably restricted by three-family unitarity.*

In the case of four standard families, the absolute square of the $4 \times 4 \mathrm{KM}$ matrix can be calculated directly with the help of Jarlskog's projection operator trace technique ${ }^{7}$

$$
\left|\left(\mathrm{V}_{K M}\right)_{\alpha \beta}\right|^{2}=\operatorname{Tr}\left(P_{\alpha} P_{\beta}^{\prime}\right)
$$

*For another somewhat Iess restrictive delermination of the extended KM matrix, sce the analysis of Gilman, Kleinknecht and Renk in re.. 13. 
where the $\alpha$ th projection operator for the up quark sector is

$$
P_{\alpha}=\frac{\left(\mathbf{M}^{U}-\lambda_{\beta} I\right)\left(\mathbf{M}^{U}-\lambda_{\gamma} I\right)\left(\mathbf{M}^{U}-\lambda_{\delta} I\right)}{\left(\lambda_{\alpha}-\lambda_{\beta}\right)\left(\lambda_{\alpha}-\lambda_{\gamma}\right)\left(\lambda_{\alpha}-\lambda_{\delta}\right)}
$$

and $\lambda_{\alpha}$, etc., are the mass eigenvalues of the up quark matrix given in (1b) with the appropriate signs; the subscripts are permutations of $1,2,3,4$. In the case of an isosinglet fourth family, we have the identity

$$
\left(\mathcal{V}_{K M}\right)_{\alpha \beta}=\left(\mathbf{V}_{K M}\right)_{\alpha \beta}-U_{\alpha 4} U_{A \beta}^{\prime \dagger}
$$

From this it follows that the square of this mixing matrix element can be written as a generalization of the Jarlskog trace technique

$$
\left|\left(\mathcal{V}_{K M}\right)_{\alpha \beta}\right|^{2}=\operatorname{Tr}\left(P_{\alpha} P_{\beta}^{\prime}-E_{4} P_{\alpha} P_{\beta}^{\prime} E_{4}-E_{4} P_{\beta}^{\prime} P_{\alpha} E_{4}+E_{4} P_{\alpha} E_{4} P_{\beta}^{\prime} E_{4}\right)
$$

which takes into account the nonunitary nature of $\mathcal{V}_{K M}$. Ifere $E_{4}=\operatorname{diag}(0,0,0,1)$.

As was previously done ${ }^{1-4}$ in the case of three-family mass matrices, the squares of the KM matrix elements are fitted to within one slandard deviation, but now only for $V_{u d}, V_{u s}, V_{c d}$ and $V_{c b}$ by varying $m_{t}, \phi_{A^{\prime}}, \phi_{B^{\prime}}, \phi_{D}$ and $\phi_{D^{\prime}}$. The results are insensitive to the two phase angles $\phi_{D}$ and $\phi_{D}$, and we can set them equal to zero. With the lighter quark masses chosen following the Gasser-I,cutwyler prescription ${ }^{14}$ as in ref. 4, the results are shown in Fig. la and $1 \mathrm{~b}$ for the fourth-family doublet quarks and isoscalar quarks, respectively, where the physical regions for the KM matrix appear as annular rings with the phase angle $\phi_{A^{\prime}}$ constrained to $85^{\circ} \pm 2^{\circ}$.

The box diagrams for the $B_{d}-\bar{B}_{d}$ mixing parameter $x_{d}$ in the three family case are proportional to the combinations $m_{l}^{2}\left|V_{t d}^{*} V_{t b}\right|^{2} R$, where $R$ is a correction factor involving logarithms of quark masses and the charged Iliggs mass in the case of two doublet Higgs models. ${ }^{2}$ From the ARGUS and CIEO data, ${ }^{15}$ one finds that this 
combination is approximately equal $1 \mathrm{o}^{2}$

$$
m_{t}^{2}\left|V_{t d}^{*} V_{t b}\right|^{2} R=(2.0 \pm 0.5) \frac{(0.140)^{2}}{B_{B} f_{B}^{2}}
$$

All three factors on the lefthand side of (9a) vary as one sweeps through the $\phi_{B^{\prime}}$ vs. $m_{t}$ plane. With the four-family models of interest in this letter, major contributions to $x_{d}$ arise from the $X$ quark, as well as crossed terms involving $t$ and $X$ exchange. Inspection of the KM matrices derived for each case by fitting the Schubert data according to the prescription described above, however, reveals that the $X$ quark exchange dominates the onc-loop graphs, especially in the isosinglet case. For simplicity, we replace (9a) by the approximation

$$
m_{X}^{2}\left|V_{X d}^{*} V_{X b}\right|^{2} R_{X}=(2.0 \pm 0.5) \frac{(0.140)^{2}}{B_{B} \int_{B}^{2}}
$$

where $R_{X}$ is evaluated for the $X$ quark rather than the $t$ quark. With $m_{X}^{\text {phys }}$ fixed at $250 \mathrm{GeV}$, we have $m_{X}=m_{X}\left(m_{X}\right)=211 \mathrm{GeV}, R_{X}=0.47$ for the minimal Iliggs model and $R_{X}=1.33$ for the two-doublet IIiggs model, where we have chosen 50 $\mathrm{GeV}$ for the charged IIiggs scalar mass and have set the two vacuum expectation values equal. Now only the square of the KM matrix elements on the lefthand side of $(9 \mathrm{~b})$ varies with $\phi_{B^{\prime}}$ and $m_{\ell}$.

The physically-allowed regions for the $B_{d}-\ddot{B}_{d}$ mixing results appear as bands, with the single-hatched regions referring to the standard Higgs model and the double-hatched regions to the two-doublet, Iliggs model. Overlap of the ring and band represents the true physical region for the model al hand. With the assignments of $m_{X}^{\text {phys }}=250 \mathrm{GeV}$ and $m_{Y}^{\text {phys }}=240 \mathrm{GeV}$, we see that the preferred top quark mass is about $70 \mathrm{GcV}$ for four standard quark families and two IIiggs doublets; with two isosinglet quarks, the preferred top mass is again about $70 \mathrm{GeV}$ but with minimal Iliggs structure, while the top mass drops to about $46 \mathrm{GeV}$ with 
two Higgs doublets. In fact, this latler possibility is ruled out by the recent lower bounds determined by the CDF, UA1 and UA2 groups. ${ }^{16}$

It is of interest to compare the predictions of other physical quantities for four families with those derived earlier for three families. This is done in Table I, where we have included only the three-family results without evolution. As shown in ref. 3, evolution from a $100 \mathrm{TeV}$ scale down to $1 \mathrm{GeV}$ changes the three family results by $5-10 \%$. The effective $J$ value associated with CP violation can again be defined by $^{17}$

$$
\begin{aligned}
J_{e f f} & \equiv \operatorname{Im}\left(V_{u s}, V_{c b} V_{u b}^{*} V_{c s}^{*}\right) \\
& \simeq\left|V_{u s}\right|\left|V_{c b}\right|\left|V_{u b}\right|\left|V_{c s}\right| \sin \delta
\end{aligned}
$$

from which the CP phase angle $\delta$ can be determined, where we have chosen the new standard parametrizalion of the KM matrix in ref. 18. The $x$, parameter for $B_{s}-\vec{B}_{s}$ mixing is approximately $x_{d}\left|V_{X}\right|^{2} /\left|V_{X d}\right|^{2}$, while the $b \rightarrow u / b \rightarrow c$ ratio again involves $\left|V_{u b}\right| /\left|V_{c b}\right|$. The bag parameter $B_{K}$ in $\mathrm{K}$ decay can again be determined from $\epsilon_{K}$, and to a good approximation is given by ${ }^{19}$

$$
B_{K} \simeq\left|\epsilon_{K}\right|\left[\frac{G_{F}^{2}}{12 \sqrt{ } 2 \pi^{2}} \frac{m_{K}}{\Delta m_{K}} f_{K}^{2} \eta_{2} m_{X}^{2} R_{X} \operatorname{Im}\left(V_{X_{s}}^{*} V_{X d}\right)^{2}\right]^{-1}
$$

Unfortunately, this result is not invariant, unlike $J$ in Eq. (10), but depends on the parametrization adopted for the phases in the KM mixing malrix. We choose $\eta_{2}=0.6$ and evaluate $B_{K}$ for the convention of ref. 16 in Table I. The results for $\epsilon^{\prime} / \epsilon$ as determined from penguin diagrams are even more complex with four families present, and have not becn evaluated.

As seen from Table 1 , most of the results are in reasonable agrement with the presently available information with the exception of the isosinglet case with two lliggs doublets, where a value of $m_{t}=16 \mathrm{GeV}$ has already been excluded. ${ }^{16}$ The bag parameter in $\mathrm{K}$ decay, as calculated in the parametrizalion of ref. 18 , tends 
to be low with four families, as alrcady noted by Gronau, Johnson and Schechter ${ }^{20}$ on the basis of a model-independent argument. We do not fecl, however, that these results are sufficient to exclude the four-family models at this time, given the parametrization noninvariance and phase uncertainty mentioned above. In fact, a relative phase rotation of the first and second columns by just $12^{\circ}$ is sufficient to restore $B_{K}$ to the preferred value near 0.66 quoted by Bardeen, Buras and Gérard in ref. 21 .

As a further comparison of the two four-family cases, we present the extended KM matrices for the spccial points in the central overlap regions of Fig. I that have been singled out in Table I. Although, in principle, one should be able to calculate all the matrix elements from their absolute values and unitarity, ${ }^{22}$ when it applies, these evaluations were carried out by exactly computing the unitary matrices $U$ and $U^{\prime}$ of (2) and then making use of Eqs. (4a,b). Finally, phase rotations of the quark fields then enable us to write $V_{K M}$ in the phase convention of ref. 18 for the fourth-doublet casc

$$
\begin{aligned}
V_{K M}^{4 t h D O U B L E T} & =\left(\begin{array}{cccc}
0.9753 & 0.2211 & -0.0004 & -0.0015 \\
-0.2205 & 0.9728 & 0.0467 & -0.0482 \\
0.0069 & -0.0265 & 0.9383 & 0.3445 \\
-0.0123 & 0.0601 & -0.3425 & 0.9373
\end{array}\right) \\
& \pm i\left(\begin{array}{cccc}
0 & 0 & 0.0027 & -0.0023 \\
0 & 0.0012 & 0 & 0.0228 \\
0 & 0.0079 & 0.0023 & 0 \\
-0.0078 & 0.0203 & 0.0003 & 0
\end{array}\right)
\end{aligned}
$$


and for the isosinglet case

$$
\begin{aligned}
V_{K M}^{2 \text { SINGLETS }} & =\left(\begin{array}{cccc}
0.9753 & 0.2210 & -0.0004 & -0.0001 \\
-0.2202 & 0.9719 & 0.0508 & -0.0109 \\
0.0039 & -0.0148 & 0.8889 & 0.0996 \\
0.0174 & -0.0778 & 0.4416 & 0.0507
\end{array}\right) \\
& \pm i\left(\begin{array}{cccc}
0 & 0 & 0.0027 & -0.0006 \\
0 & 0.0016 & 0 & 0 \\
0.0008 & 0.0001 & 0 & 0 \\
0.0040 & 0.0008 & 0 & 0
\end{array}\right)
\end{aligned}
$$

It is clear from these matrices that, while $V_{K M}$ is unitary in the former case and nonunitary in the latter case, in both cases some elements in the fourth row are larger than their counterparts in the third row. This plays a major role in the successful fit to the $B-\bar{B}$ mixing data given in Fq. (9b) but negates the simplifying procedure of Bjorken ${ }^{23}$ to extract the phases of the KM matrix elements from their absolute values. In both cases, we find that the ratio $\left|V_{u b}\right| /\left|V_{c b}\right|=0.053$, as in the three family Fritzsch model with two Ifiggs doublets. ${ }^{2}$ It is also clear that the $3 \times 3$ submatrices for the three observed families are not greatly different from that of (6), deduced by Schubert from present dala and three family unitarity; however, we note that the fourth row and fourth column are considerably more asymmetric than the first three, especially with isosinglets present.

Of special interest in the isoscalar model is the appearance of trec-level flavorchanging neutral currents (FCNC's). The neutral current in this case is given by

$$
\begin{aligned}
J_{\mu}^{(0)} & =\bar{\psi}_{L}^{U} \gamma_{\mu} \psi_{L}^{U}-\bar{\psi}_{L}^{D} \gamma_{\mu} \psi_{L}^{D}-2 \sin ^{2} \theta_{W} J_{\mu}^{e m} \\
& =\bar{\Psi}_{L}^{U} \gamma_{\mu} V_{N C}^{U} \Psi_{L}^{U}-\bar{\Psi}_{L}^{D} \gamma_{\mu} V_{N C}^{D} \Psi_{L}^{D}-2 \sin ^{2} \theta_{W} J_{\mu}^{e m}
\end{aligned}
$$

where in (13a) only the three weak doublet ficlds enter, while the four up and 
down mass eigenficlds appear in (13b). The neutral current mixing matrices for the lefthanded currents are related to the $4 \times 4$ unitary matrices $U$ and $U^{\prime}$ in (2) by

$$
\begin{aligned}
& \left(V_{N C}^{U}\right)_{\alpha \beta}=U_{\alpha i}\left(U^{\dagger}\right)_{i \beta}=\delta_{\alpha \beta}-U_{\alpha 4}\left(U^{\dagger}\right)_{4 \beta} \\
& \left(V_{N C}^{D}\right)_{\alpha \beta}=U_{\alpha i}^{\prime}\left(U^{\prime \dagger}\right)_{i \beta}=\delta_{\alpha \beta}-U_{\alpha 4}^{\prime}\left(U^{\prime \dagger}\right)_{4 \beta}
\end{aligned}
$$

In the phase convention where $V_{N C}^{U}$ is taken to be real and for the parameters of the isoscalar model in the center of the physical region in Fig. $1 \mathrm{~b}$ where $m_{t}=70.5$ $\mathrm{GeV}$, the explicit forms of these two FCNC malrices, are calculated to be

$$
\begin{gathered}
V_{N C}^{U}=\left(\begin{array}{rrrr}
1.0000 & -0.0002 & 0.0015 & 0.0030 \\
-0.0002 & 0.9958 & 0.0289 & 0.0576 \\
0.0015 & 0.0289 & 0.8002 & -0.3988 \\
0.0030 & 0.0576 & -0.3988 & 0.2040
\end{array}\right) \\
V_{N C}^{D}=\left(\begin{array}{rrrr}
1.0000 & -0.0001 i & 0.0004 i & 0.0037 i \\
0.0001 i & 0.9997 & 0.0018 & 0.0160 \\
-0.0001 i & 0.0018 & 0.9877 & -0.1104 \\
-0.0037 i & 0.0160 & -0.1104 & 0.0126
\end{array}\right)
\end{gathered}
$$

Note that the $\bar{c} Z$ and $\bar{t} c Z$ couplings are at the level of 0.03 and suggest that the FCNC $Z$ decay, $Z \rightarrow \bar{c} t+\bar{t} c$, may be observable in the future at ILP and SLC. The large value for the $\vec{X} t Z$ and $\bar{X} X Z$ couplings suggests that the $X \rightarrow t Z$ decay will compete favorably with the $X \rightarrow Y W$ decay, as scen from (15b) and (12b).

By starting from the Fritzsch form of the mass matrices extended to four standard quark families or to three families plus a pair of isosinglet quarks, we have studied the viability of these two models in explaining both the "observed" KM mixing matrix and large $B_{d}-\bar{B}_{d}$ mixing. For this purpose, we have imposed restrictions only on the accurately known $V_{u d}, V_{u s}, V_{c d}$ and $V_{c b}$ elements of the KM mixing matrix. We have also set the heavy quark masses at $m_{X}=250 \mathrm{GeV}$ and $m_{Y}=240$ 
$\mathrm{GeV}$, comparable to the infrared fixed points, so as to minimize radiative corrections to the $\rho$ parameter and renormalization effects on the mass matrices which will be reported elsewhere. Our results indicate that, for either standard model extension, good agreement can be obtained with a top quark mass of $m_{t} \simeq 70 \mathrm{GcV}$, if two Higgs doublets are used for the four doublet version and just minimal IIiggs structure for the isosinglet version. The flavor-changing neutral current matrices appearing in the isosinglet case are of considerable interest in rarc $Z$ decay studies at the near-operational $e^{+} e^{-}$colliders. The $70 \mathrm{GcV}$ top mass is to be contrasted with our previous result of 94 or $91 \mathrm{GeV}(88 \mathrm{GeV}$ with renormalization effects included) in the presence of just three standard doublet quark families. If we drop the heavy quark masses to $150 \mathrm{GeV}$ and $140 \mathrm{GcV}$, respectively, we find the top quark mass can be lowered to approximately $60 \mathrm{GeV}$. This suggests rather strongly that the appearance of a top quark mass significantly lower than $90 \mathrm{GeV}$ can be a strong hint of new physics beyond the standard model, as we now know it.

The author thanks Joln Ellis and the CERN 'TH Division for its kind hospilality while this rescarch was in progress. He also wishes to acknowledge useful conversations with Manfred Lindner, Guido Altarclli and André Martin on various aspects of this work. This research was supported in part by Grant No. PIIY-870120 from the U. S. National Science Foundation. 


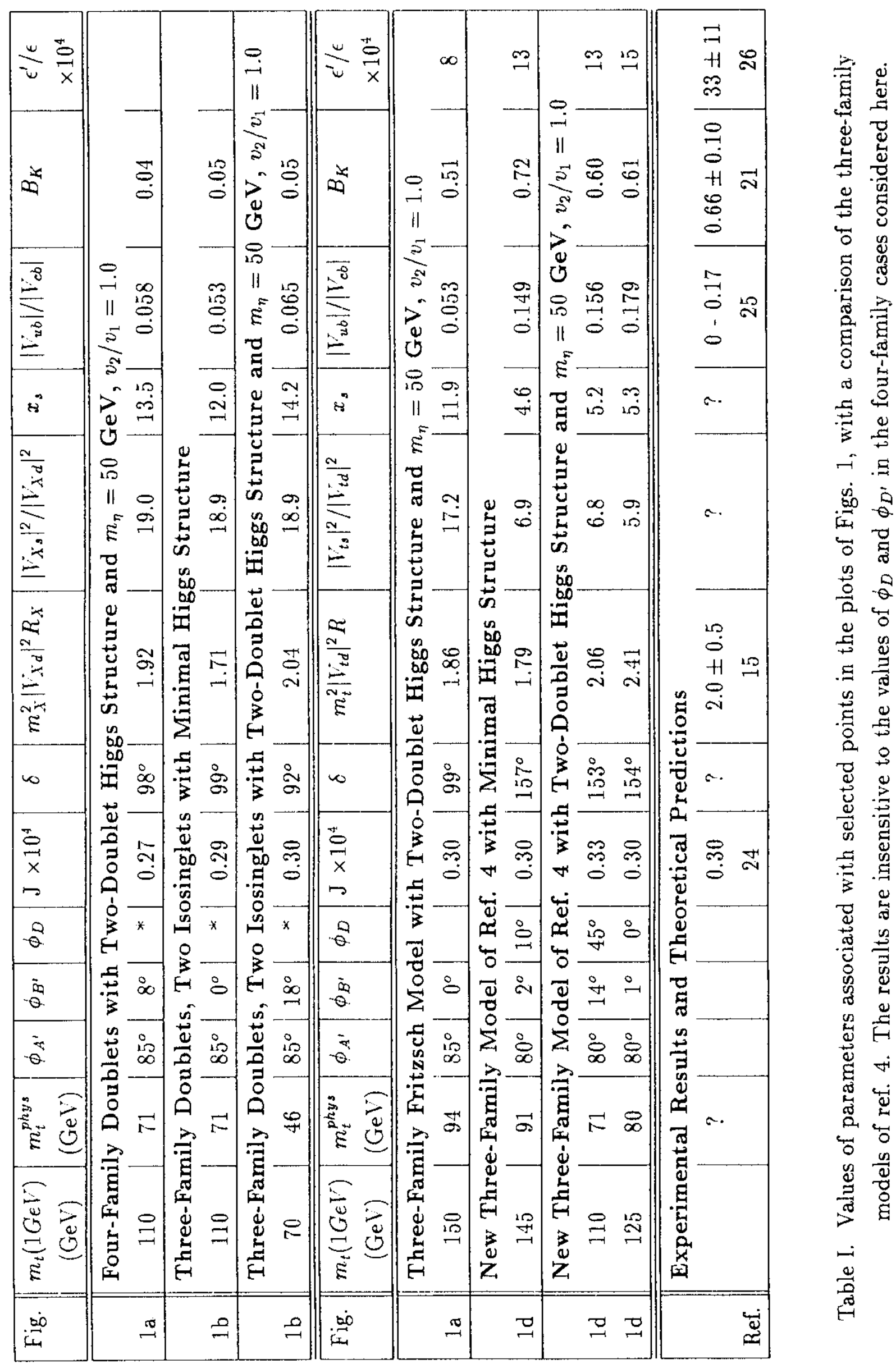




\section{References}

[1] C. H. Albright, C. Jarlskog and B.- $\AA$. I,indholm, Phys. Lett. B 199 (1987) 553.

[2] C. H. Albright, C. Jarlskog and B.- $\AA$. Iindholm, Phys. Rev. I) 38 (1988) 872.

[3] C. H. Albright and M. L. Lindner, Phys. Rev, Lett. B 213 (1988) 347, and manuscript FNAL-Conf-88/76-'T submitied to the XXIV International Conference on High Energy Physics (Munich, 1988).

[4] C. H. Albright and M. L. Lindner, Fermilab preprint PUB-89/17-'T, to be published.

[5] H. Fritzsch, Phys. Lett. B 70 (1977) 136; B 73 (1.978) 317; B 166 (1986) 423.

[6] H. Fritzsch, ref. 5; 'T. Kitazoe and K. Tanaka, Phys Rev. D 18 (1978) 3476; II. Georgi and D. V. Nanopoulos, Phys. Lett. B 82 (1979) 392 and Nucl. Phys. B 155 (1979) 52; L. F. Li, Phys. Let.t. B 81B (1979) 461; A. A. Davidson, Phys. Lett. B 122B (1983) 112; B. Stech, Phys. Lelt. B 1.30 (1983) 189; M. Shin, Phys. Ielt. B 145 (1984) 285; M. Shin, in Proceedings of the TwentyFirst Rencontre de Moriond, "Progress in Electroweak Interactions," Ed. J. Tran Thanh Van (Editions Frontic̀res, 1986); T. P. Chang and I.-F. Li, Phys. Rev. D 34 (1986) 219; T. Wollenstein, Phys. Rev. I) 34 (1986) 897; J.-M. Gérard, B. Grzadkowski and M. Lindner, Phys. Lett. B 189 (1987) 453; B. Bijnens and C. Wetterich, Phys. I,ett. B 199 (1987) 525; II. IIarari and Y. Nir, Phys. Lett. B 195 (1987) 586; Y. Nir, Nucl. Phys. B 306 (1988) 14.

[7] C. Jarlskog, Phys. Rev. D 35 (1987) 1685; C. Jarlskog and A. Kleppe, Nucl. Phys. B 286 (1987) 245; C. Jarlskog, Phys. R.ev. D) 36 (1987) 2128. 
[8] I. I. Bigi, Z. Phys. C 27 (1985) 303; M. Gronau and J. Schechter, Phys. Rev. D 31 (1985) 1668 and Phys. Rev. D 33 (1986) 2641; A. A. Anselm, J. L. Chkareuli, N. G. Uraltsev and T. A. Zhukovskaya, Phys. Lett. 156B (1985) 102; X.-G. Ile and S. Pakvasa, Phys. I,et. 156 B (1985) 236; U. Turke, IS. A. Paschos, H. Usler and R. Decker, Nucl. Phys. B 285 (1985) 313; K. Kang and M. Shin, Phys. Lett. 165B (1985) 383; M. Shin, R. S. Chivukula, and J. M. Flynn, Nucl. Phys. B 271 (1986) 509; 'T. Iayashi, M. Tanimoto and S. Wakaizumi, Prog. 'Theor. Phys. 75 (1986) 353; 'T. P. Cheng and L.-F. Li, Phys. Rev. Lett. 55 (1985) 2249 and Phys. Rev. D 34 (1986) 226; X.-G. He and S. Pakvasa, Nucl. Phys. B 278 (1986) 905; R. Johnson, J. Schechter and M. Gronau, Phys. Rev. D 33 (1986) 2641; M. Tanimoto and A. Takatsu, Phys. Rev. D 34 (1986) 1474; M. Tanimoto, 'T. Ilayashi, R. Najima and S. Wakaizumi, Prog. Theor. Phys. 76 (1986) 1098; K. S. Babu, Phys. Rev. D 35 (1987) 3477; D.-d. Wu and Y.-I. Wu, Chin. Phys. Lett. 4 (1987) 111; M. Tanimoto, Y. Suctake and K. Senba, Phys. Rev. D) 36 (1987) 2119 and \%. Phys. C 40 (1988) 539; W.-S. Hou and A. Soni, Phys. Jett. B 196 (1987) 92.

[9] B. S. Balakrishna, Phys. Rev. Lett. 60 (1988) 1602; B. S. Balakrishna, A. Г. Kagan and R. N. Mohapatra, Phys. Lett. B 205 (1988) 345; A. L. Kagan, U. of Maryland preprint, UMDEPP 89-109, to be published.

[10] B. Grzadkowski and M. Lindner, Phys. Iett. B 193 (1987) 71; B. Grzadkowski, M. Lindner and S. Theisen, Phys. Lelt. B 198 (1987) 64.

[11] M. Veltman, Nucl. Phys. B 123 (1977) 89.

[12] K. R. Schubert, in Proccedings of the 1987 PPS Conference (Uppsala, 1987).

[13] F. J. Gilman, K. Kleinknecht and B. Renk, Review of Particle Properties, Parlicle Data Group, Phys. Ielt. B 204 (1988). 
[14] J. Gasser and H. Leutwyler, Phys. Rep. C 87 (1982) 77.

[15] ARGUS Collab., H. Albrecht, et al., Phys. Lett. B 209 (1988) 119; CleO Collab., C. Bebek et al., in Procecdings of the XXIV International Conference on High Energy Physics (Munich, 1988).

[16] CDF, UA1 and UA2 groups, in Procedings of the Twenty-Fourth Rencontre de Moriond and the Ia Thuile Conference, 1989.

[17] C. Jarlskog, Phys. Rev. Iett. 55 (1985) 1039; Zcit. Phys. C 29 (1985) 491.

[18] L. L. Chau and W. Y. Keung, Phys. Rev. T.ett. 53 (1981) 1802; II. Harari and M. Leurer, Phys. Lett. B 181 (1986) 123; H. Fritzsch, Phys. Iett. B 189 (1.987) 191 .

[19] T. Inami and C. S. Lim, Prog. Theor. Phys. 65 (1981) 297; J. S. Magelin, Phys. Rev. D 23 (1981) 119; F. J. Gilman and M. B. Wise, Phys. Rev. D 27 (1983) 1128 .

[20] M. Gronau, R. Johnson and J. Schechter, Phys. Lett. B 201 (1988) 151.

[21] A. J. Buras and J.-M. Gérard, Nucl. Phys. B261 (1986) 371; W. A. Bardeen, A. J. Buras and J.-M. Gérard, Phys. Lett. B 211 (1988) 343.

[22] G. C. Branco and T.Lavoura, Phys. Lett. B 208 (1988) 123; C. Jarlskog and R. Stora, Phys. Lett. B 208 (1988) 268; $\Lambda$. Martin, private communication (to be published); G. Aubcrson, Phys. Lett. B 216 (1989) 167; L. Lavoura, Lisbon preprint, IFM-1./89, to be published.

[23] J. D. Bjorken, Fermilab preprint PUB-88/121-T, to be published.

[24] J. F. Donoghue, 'T. Nakada, F. A. Paschos and D. Wyler, Phys. Lett. B 195 (1.987) 285. 
[25] ARguS Collab., II. Albrecht et al., Phys. Lett. B 209 (1988) 119; CT,EO Collab., C. Bebck et al., Phys. Rev. I,ctt. 62 (1989) 8.

[26] H. Burkhardt et al., Phys. Lett. B 206 (1988) 1169; M. Woods et al., Phys. Rev. Lett. 60 (1988) 1695.

\section{Figure Caption}

Figure 1: Phase angle $\phi_{B^{\prime}}$ vs. $m_{\ell}(1 G e V)$ and $m_{t}^{\text {phys }}$ plots showing the physicallyallowed KM matrix annular rings and $B_{d}^{o}-\bar{B}_{d}^{o}$ mixing bands, the latter single-hatched for the standard Iliggs model and double-hatched for the two-doublet Itiggs model. Here (a) refers to the four doublet model and (b) to the three doublet plus two isosinglet model. The masses for the fourth-family heavy quarks have been set at $250 \mathrm{GeV}$ for the charge $2 / 3 X$ quark and $240 \mathrm{GcV}$ for the charge - 1/3 Y quark. Equal VEV's have been used for the double lliggs model with $50 \mathrm{GeV}$ for the charged Higgs mass. 


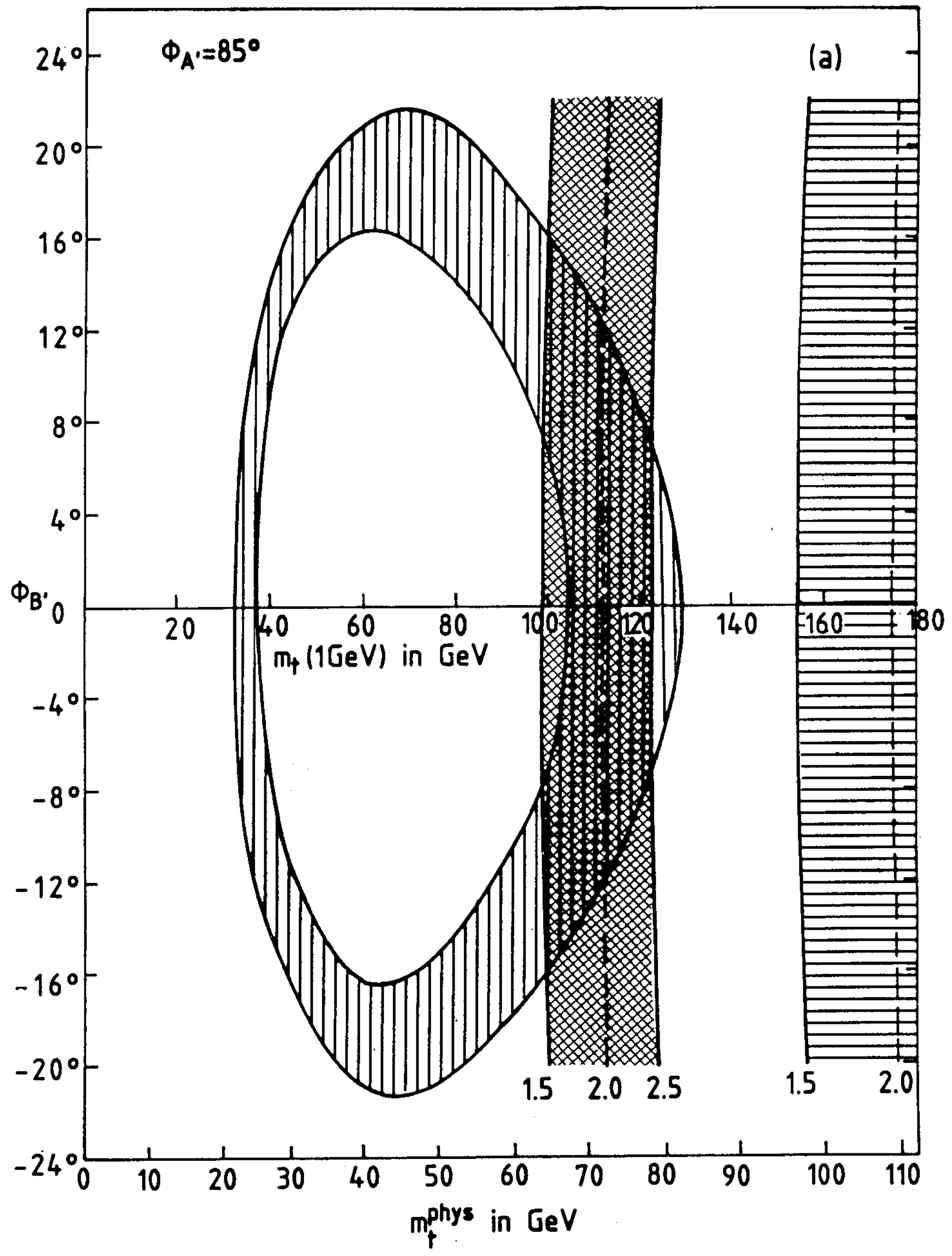




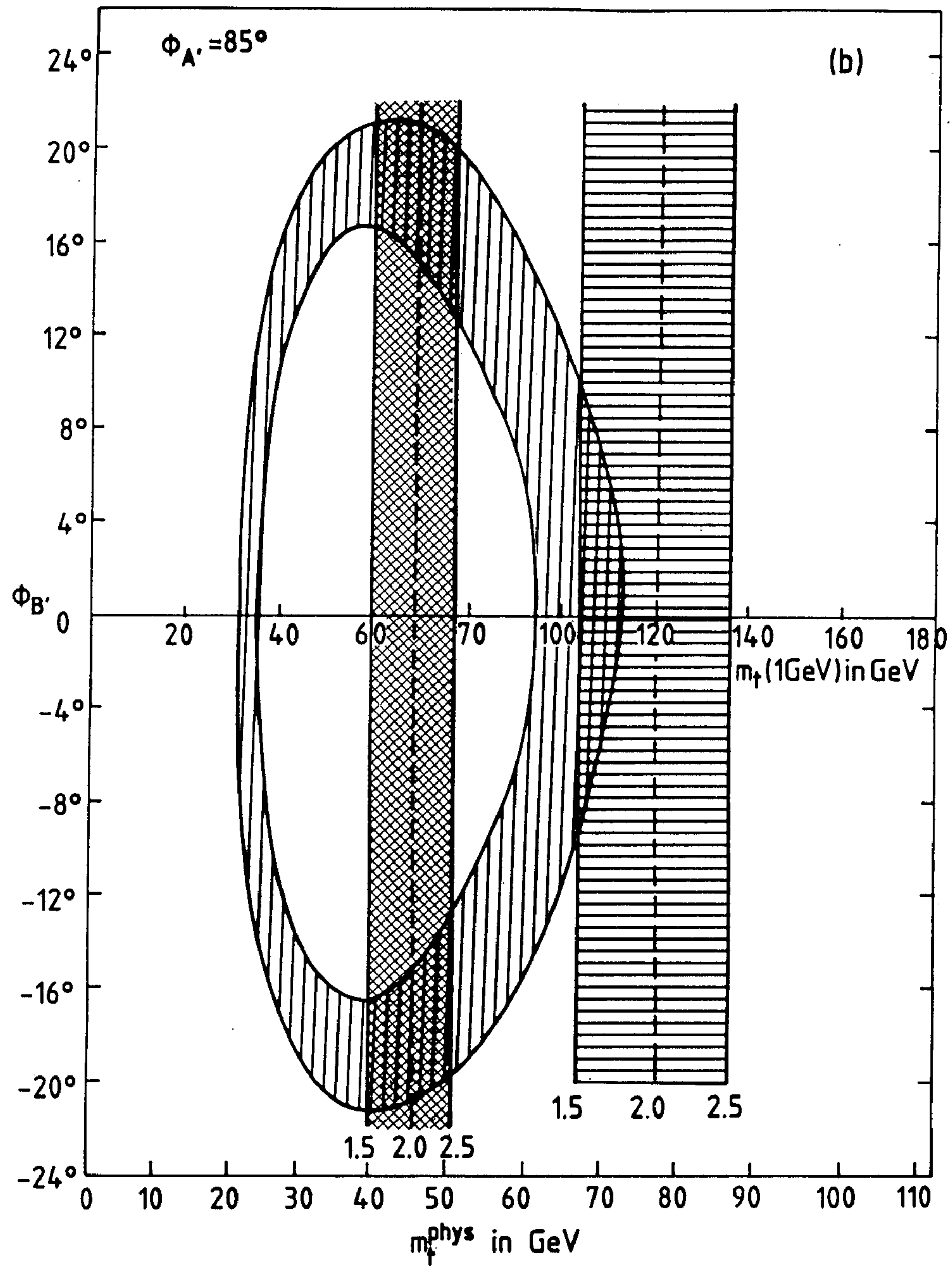

